\title{
Peces endémicos de sistemas fluviales de la Selva Atlántica en la Argentina: Áreas prioritarias para su conservación
}

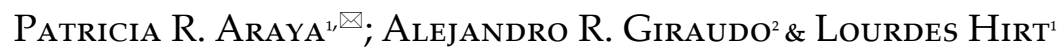 \\ ${ }^{1}$ Laboratorio Ictiofauna, Facultad Ciencias Exactas Químicas y Naturales, Universidad Nacional de Misiones. ${ }^{2}$ Instituto \\ Nacional de Limnología, Laboratorio de Biodiversidad y Conservación de Tetrápodos (CONICET, UNL).
}

\begin{abstract}
RESUMEN. Los ecosistemas de agua dulce están entre los más amenazados por las actividades humanas y el cambio climático, por lo cual es prioritario conservarlos. La provincia de Misiones, Argentina, tiene la tasa de endemismo más alta de todas las regiones ícticas de la Argentina. El objetivo de este trabajo fue establecer el estado y la prioridades de conservación de los cursos fluviales interiores de la Selva Atlántica en la Argentina en función de la presencia de peces de distribución endémica en Misiones, evaluando su representatividad en el sistema de áreas protegidas y determinando vacíos de conservación. Se analizó la integridad ecológica de los sistemas fluviales según el grado de modificaciones, calculado mediante el índice rápido para la evaluación de calidad de ecosistemas de ribera (QBR). Se estableció su grado de protección mediante el porcentaje del curso fluvial fuera, marginal o dentro de las áreas protegidas. Se priorizaron los arroyos que eficientemente incluyeron a todas las especies endémicas. Treinta y seis cursos de agua albergan 79 especies de peces endémicos, de los cuales 22 arroyos resultan prioritarios para su conservación porque podrían proteger a todos los peces endémicos. Sin embargo, más de la mitad de estos arroyos no poseen protección y sufren alteraciones como destrucción de la vegetación marginal, represamientos, polución e introducción de especies exóticas. La mayor prioridad de conservación la tienen los cursos de agua Iguazú, Yabotí y Urugua-í, con sus afluentes.
\end{abstract}

[Palabras clave: ictiofauna neotropical, endemismo, arroyos, Misiones]

Abstract. Priority areas for conservation: Endemic fishes of the Atlantic Forest fluvial system in Argentina. Freshwater ecosystems are among the most threatened by human activities and climate change; so, their conservation is a priority. The province of Misiones has the highest rate of fish endemisms of all the ictic areas of Argentina. Our objective was to establish the status and conservation priorities of the interior river courses of the Atlantic Forest in Argentina in relation to the presence of endemic and distribution-restricted fish in Misiones, evaluating their representativeness in the system of protected areas and determining gaps in conservation. The ecological integrity of the river systems was analyzed according to the degree of modifications calculated by means of the rapid index for the evaluation of the quality of riverside ecosystems (QBR). Their degree of protection was estimated by the percentage of the river course outside, inside or marginal to protected areas. Streams that efficiently include all endemic species were prioritized. Thirty-six watercourses are home to 79 endemic fish species; 22 streams appear a priority for conservation because they would protect all endemic species. However, more than half of these streams are not currently protected and suffer alterations such as destruction of marginal vegetation, dams, pollution and introduction of exotic species. The highest priority for conservation was assigned to the Iguazú, Yabotí and Urugua-í watercourses with their tributaries.

[Keywords: neotropical ichthyofauna, endemism, streams, Misiones]

\section{INTRODUCCIÓN}

La gran presión antrópica que se ejerce sobre los recursos naturales limita la recuperación natural de los ecosistemas; uno de sus efectos es la pérdida acelerada de biodiversidad. Los ecosistemas acuáticos continentales se encuentran entre los sistemas más biodiversos del planeta y entre los más amenazados por el uso intensivo que el ser humano hace del agua y del territorio. Las principales causas de su perturbación son: 1) la pérdida, degradación y fragmentación del hábitat, 2) el deterioro de la calidad del agua (i.e., contaminación y eutrofización), 3) la sobreexplotación del recurso mediante el embalsado o la extracción, y 4) la introducción de especies

Editor asociado: Fernando Milesi exóticas (Pacific Rivers Council 2007). Entre los grupos más representativos de los ecosistemas acuáticos se encuentran los peces de agua dulce, que representan un cuarto del total de la biodiversidad de vertebrados del planeta (Abell 2002) y se hallan en grave estado de conservación con un $30 \%$ de las especies incluidas en alguna de las categorías de amenaza de la UICN (WWF 2010; Closs et al. 2016).

La Región Neotropical contiene una de las mayores concentraciones de biodiversidad en el planeta. En particular, sus más de 6025 especies de peces $(\sim 10 \%$ de todos los vertebrados) representan la mayor 
diversidad de peces en el mundo (Albert and Reis 2011). La cuenca del Plata es la segunda más extensa de Sudamérica y la quinta a nivel mundial, con $\sim 1500$ especies de peces incluyendo endemismos en especial notorios en las áreas de tierras altas como las cuencas del río Iguazú y los arroyos de mesetas basálticas del sudeste de Brasil y el nordeste de la Argentina (Albert and Reis 2011), donde se encuentran varias especies amenazadas (Barletta et al. 2010; Baigún et al. 2012; Casciotta et al. 2016). La provincia de Misiones incluye una porción de la región de la Selva Atlántica (también denominada Selva Paranaense o Selva Misionera en la Argentina), la segunda mayor ecorregión de pluviselva del continente americano, considerada uno de los cinco hotspots más amenazados del mundo (Myers et al. 2000). Los ecosistemas acuáticos de la provincia de Misiones, clasificados biogeográficamente dentro de la Provincia de los Grandes Ríos (López et al. 2008), poseen una ictiofauna notablemente diversa con representación del $50 \%$ de las 570 especies de peces continentales de la Argentina y especies endémicas de los arroyos de Misiones (Casciotta et al. 2016; Mirande and Koeber 2020), así como de otros grupos muy dependientes de los humedales, como anfibios y reptiles (Giraudo 2017). En los últimos años, la descripción de numerosas especies de peces en los cursos interiores de Misiones (Azpelicueta et al. 2004; Miquelarena and López 2004; Casciotta and Almirón 2008; Almirón et al. 2014a,b; Pialek et al. 2015) permitió actualizar y dar a conocer la lista de especies endémicas.

Giraudo (2017) sintetiza la información disponible sobre humedales de Misiones e indica como sus principales amenazas a las represas y a la deforestación de la Selva Paranaense. Las represas como Yacyretá y Urugua-í, y otras proyectadas como Garabí, Roncador y Corpus, representan profundos cambios ecológicos para los ríos y arroyos, incluyendo: 1) la pérdida de la mayoría de los humedales naturales y de otros hábitats aledaños, la alteración de variaciones hidrológicas naturales y propiedades físicoquímicas del agua (eutrofización acelerada, acidificación, estratificación térmica), y 2) alteraciones profundas de las comunidades biológicas, incluyendo cambios en las comunidades ícticas por la presencia de barreras para las migraciones y el aumento de las invasiones biológicas (i.e., peces como los de los géneros Tilapia, Geophagus e Ictalurus han invadido las cuencas de Urugua-í y del Iguazú superior, amenazando a las especies autóctonas) (Gómez et al. 2009; Baigún et al. 2011). Por lo menos tres especies de caracoles acuáticos de correderas (Aylacostoma) se extinguieron en sus hábitats naturales como consecuencia de la represa de Yacyretá, donde eran endémicos, y otra especie del género subsistió una única población con baja diversidad genética (Vogler et al. 2015). Un proyecto de trasvasar agua desde el Iguazú hacia la represa de Urugua-í para aumentar el caudal y proveer más energía significaría graves problemas para las especies endémicas de ambos ríos y un impacto indeseable en áreas donde los recursos naturales sustentan un turismo creciente. Las producciones agrícolas que requieren deforestación son las plantaciones de pinos y eucaliptos, los cultivos de tabaco, té y yerba, y las pasturas implantadas para realizar ganadería; a estas actividades se le suma la creciente urbanización. La deforestación disminuye la retención del agua, acelera el drenaje superficial y aumenta el arrastre de sedimentos, lo que promueve la erosión y la pérdida de nutrientes de los suelos, y modifica los ciclos hidrológicos naturales y las características físico-químicas del agua (Giraudo 2017). Mantener la biodiversidad implica conservar la composición, la estructura y las funciones de los paisajes, los ecosistemas, las comunidades, las poblaciones, las especies y la información genética en diversas escalas temporales y espaciales (Galindo-Leal 2000). En el caso de los ríos, el estado de conservación de la vegetación riparia y de la región de captación de su cuenca es un atributo esencial para considerar en su conservación (Nel et al. 2007). La Selva Atlántica juega un rol importante en la conservación de las cuencas hídricas, asegurando la cantidad y la calidad de agua esenciales para conservar la ecorregión de los ríos y arroyos del Alto Paraná, una ecorregión Global 200 de agua dulce (Di Bitetti et al. 2003).

Una de las principales respuestas a la necesidad de conservación de la biodiversidad es crear áreas protegidas. En las últimas décadas se desarrollaron estrategias complejas para proteger la biodiversidad (Margules and Pressey 2000; Sarkar and Illoldi-Rangel 2010), y se propusieron algoritmos científicos que priorizan áreas o regiones en base a información sobre diferentes aspectos (Brooks et al. 2006). Ejemplos de estos aspectos son la viabilidad o la congregación de especies focales 
(amenazadas, con distribución restringida en áreas de endemismos, características de biomas y aves gregarias) para determinar las áreas importantes para la conservación de aves (AICAs) (Di Giacomo and Spitznagel 2007) o la identificación de áreas insustituibles y vulnerables basados en la distribución y composición de las especies para identificar las áreas claves de biodiversidad (KBAs) (Eken et al. 2004). Varios de los criterios utilizados para seleccionar áreas de conservación están muy relacionados con especies endémicas y áreas de endemismo (Brooks et al. 2006; Giraudo and Arzamendia 2018). Una de las estrategias de conservación más difundida en el mundo es la identificación de hotspots o puntos calientes prioritarios para conservar la biodiversidad, definidos por Myers et al. (2000) mediante dos criterios: 1) regiones con elevada cantidad de endemismo de especies, y 2) regiones con pérdida de hábitat superior a $70 \%$. Las estimaciones de la biodiversidad mundial suelen omitir las especies de agua dulce (Myers et al. 2000; Brooks et al. 2006), a pesar de la clara evidencia de que están muy amenazados y de que los puntos calientes terrestres no siempre se superponen con los puntos calientes de agua dulce. Asimismo, los algoritmos formales para diseñar áreas protegidas rara vez utilizaron especies de agua dulce (Strayer and Dudgeon 2010). La Argentina cuenta con 493 áreas protegidas, con una superficie total aproximada de 36.462.613 ha $(13.06 \%$ del territorio nacional), mientras que la provincia de Misiones tiene 79 áreas protegidas (pocas de jurisdicción nacional, la mayoría provinciales y algunas privadas) (Giraudo et al. 2003a), con una superficie total de 456.810 ha (SiFAP 2019).

En este contexto, resulta urgente la conservación de los ambientes acuáticos para evitar la afectación de las especies de peces endémicas de la provincia de Misiones en la Argentina. Los objetivos del presente trabajo son: 1) establecer prioridades de conservación de los cursos fluviales interiores de Misiones, usando como indicadores a sus especies de peces endémicos; 2) analizar el grado de protección que poseen los peces endémicos en el sistema actual de áreas protegidas y determinar cuáles son los vacíos de conservación, y 3) ofrecer un protocolo para establecer prioridades de conservación de cursos fluviales, una aproximación poco desarrollada respecto de las estrategias de conservación de ecosistemas terrestres.

\section{Materiales y Métodos}

\author{
Área de estudio
}

La provincia de Misiones está ubicada entre los paralelos $25^{\circ} 28^{\prime}$ y $28^{\circ} 10^{\prime} \mathrm{S}$ y los meridianos $53^{\circ} 38^{\prime}$ y $56^{\circ} 03^{\prime} \mathrm{O}$. Se trata de un territorio con un cordón dorsal basáltico de tipo relictual sobre meseta (Macizo de Brasilia), constituido por las sierras de San José, del Imán y de Misiones, con su extremo norte, la de Santa Victoria. Sobre las estribaciones de este cordón nace toda la red fluvial que drena hacia los colectores principales de la región: el río Iguazú al norte, el río Paraná al oeste y el río Uruguay al este. La disposición hidrográfica de tipo periférico hace que Misiones sea un distrito particular, con innumerables cursos de agua propios (IPEC 2015). De los $~ 800$ arroyos que nacen y terminan en el mismo territorio provincial, 120 arroyos desembocan en el río Iguazú (T.IGUZ), cerca de 270 son tributarios del río Paraná (el sector denominado en este trabajo como T.PAR) y 400 corren hacia el río Uruguay (T.URU). El perfil longitudinal de los arroyos se repite en casi todos los casos: al remontar un arroyo, en sus primeros $5-10 \mathrm{~km}$, su pelo de agua está directamente influido por el gran emisario y luego comienza a entrar en un cañón zigzagueante, rocoso y estrecho, con laderas cubiertas de selva en galería. Siguiendo aguas arriba llegamos al tramo de los largos rápidos y cascadas pequeñas y medianas que se desarrollan hasta alcanzar la cota de los colectores de la meseta superior (Secretaría de Minería de la Nación 2012; Araya et al. 2013). Las particularidades geomorfológicas de los cursos fluviales de poseer cascadas y saltos (e.g., las conocidas cataratas del Iguazú) impidieron la colonización de grandes peces depredadores de los grandes ríos Paraná y Uruguay, y constituyen una de las causas del elevado grado de endemismos en su ictiofauna (Garavello et al. 1997; Ŕíčan et al. 2018; Alonso et al. 2019).

\section{Métodos}

Se confeccionó una lista de especies de peces registradas en Misiones, a fin de determinar sus distribuciones y los arroyos que ocupan. Para clasificarlos en órdenes y familias se siguió la propuesta por Nelson et al. (2016) y Reis et al. (2003). Además, se consultaron listas actualizadas de especies disponibles en Sudamérica (Reis et al. 2003), en países limítrofes (Uruguay: Oyakawa and Mattox 2009; Litz and Koerber 2014; 
Paraguay: Koerber et al. 2017) y en la Argentina (Mirande and Koerber 2020). Para determinar la distribución y el estatus de las especies de peces consideradas se consultó una amplia serie de trabajos publicados en las últimas dos décadas (López et al. 2005, 2008; Casciotta and Almirón 2008; Araya et al. 2009, 2012; Piálek et al. 2011, 2015, 2019; Říčan et al. 2011, 2017, 2018; Baumgarter et al. 2012; Almirón et al. 2014a,b; Azpelicueta and Koerber 2015; Casciotta et al. 2015, 2016, 2017; Liotta 2016; Aguilera et al. 2017; Terán et al. 2017; Alonso et al. 2019; Ferrer and Malabarba 2020; Flores et al. 2020; Mirande and Koerber 2020). Se seleccionaron las especies endémicas de cursos fluviales de la Selva Atlántica por su importancia reconocida en la conservación (Burlakova et al. 2011). Una especie endémica se caracteriza por poseer una distribución espacial limitada (Burlakova et al. 2011). Se seleccionaron aquellas especies endémicas que tienen una distribución limitada a la provincia de Misiones (i.e., no habitan en otro territorio) o bien que, además de Misiones, se limitan a arroyos de áreas cercanas del este del Paraguay, el sudeste de Brasil y el norte de Uruguay, dentro de la ecorregión de la Selva Atlántica. Todas son especies que en la Argentina sólo se registran en la provincia de Misiones. En este trabajo no se incluyeron las especies de peces que habitan Misiones, pero que poseen una distribución más amplia en la Argentina o están representadas en otras ecorregiones.

Para evaluar el estado y vacíos de conservación de los cursos de agua en relación con su ictiofauna endémica y determinar los arroyos prioritarios para la conservación se consideró el estado y el grado de protección de los ecosistemas fluviales y su cuenca.

Estado de los sistemas fluviales. Los cursos de agua fueron clasificados de acuerdo con su integridad ecológica, y se describió el grado en que fue modificada por las actividades humanas por medio del índice rápido para la evaluación de la calidad de los ecosistemas de ribera (QBR) propuesto por Munné et al. (1998, 2003). El índice QBR es la sumatoria de cuatro puntajes parciales, varía de 0-100 y se categoriza en A ( $\geq 95$ : calidad muy buena, estado natural), B (75-90: calidad buena, mayormente natural), C (55-70: calidad intermedia, inicio de alteración importante), D (30-50: alteración fuerte, mala calidad), y E ( $\leq 25$ : degradación extrema, calidad pésima). Los cuatro atributos considerados (cada uno de ellos con una puntuación parcial entre
0 y 25) fueron: 1) porcentaje de cobertura de la vegetación ribereña, 2) estructura de la cobertura, 3) calidad de la cobertura, y 4) grado de naturalidad del canal fluvial [ver Material Suplementario]. Los datos para evaluar los atributos del índice rápido se obtuvieron de: a) imágenes satelitales (Google 2020), b) registros in situ recopilados durante la presente investigación en el arroyo Paraíso (afluente río Uruguay) y en el arroyo Piray Miní (tributario río Paraná), y c) otros trabajos realizados en arroyos de Misiones (Flores et al. 2009, 2020; Araya et al. 2012, 2013; Araya, observaciones personales). Además, la estructura de la vegetación ribereña se comprobó con observaciones personales en la zona de desembocadura de los arroyos siguiendo la propuesta de Rodríguez et al. (2005), Srur (2006) y Drozd et al. (2010)

Grado de protección. Para determinar el grado de protección de los arroyos con especies de peces endémicos se siguió la propuesta de $\mathrm{Nel}$ et al. (2007), que lo estima a partir de la proporción de la superficie del curso de agua que transcurre dentro de un área protegida, que es uno de sus límites o que está por fuera de un área natural protegida (ANP). A cada arroyo se le asignó un nivel de protección de la siguiente manera: no protegido $(0 \%)$, escasamente protegido $(0.01-$ $19.99 \%)$, relativamente protegido $(20-49.99 \%)$, moderadamente protegido (50-79.99\%) y bien protegido (80-100\%). La información para estimar la proporción de cada arroyo protegido se obtuvo mediante imágenes satelitales y mapas que combinan el curso de los arroyos con una capa de áreas protegidas de Misiones cedida por el Ministerio de Ecología y Recursos Naturales Renovables (2019). Sólo se consideraron como áreas protegidas a aquellas que corresponden a áreas de tipo I y II según IUCN (protección estricta) (Dudley 2008), ya que los demás tipos de áreas protegidas pueden estar sometidas a modificaciones que afecten a los ecosistemas acuáticos (Figura 1).

Arroyos prioritarios. Para proponer arroyos prioritarios para la conservación buscando proteger todas las especies endémicas en la menor cantidad de arroyos (eficiencia máxima) se seleccionó primero el arroyo con mayor número de especies y se fueron eliminando de la matriz todas las especies presentes en el mismo. Luego, con los datos restantes, se volvió a elegir el arroyo más rico en especies y se repitió el procedimiento de eliminar las especies ya representadas y así hasta llegar a 


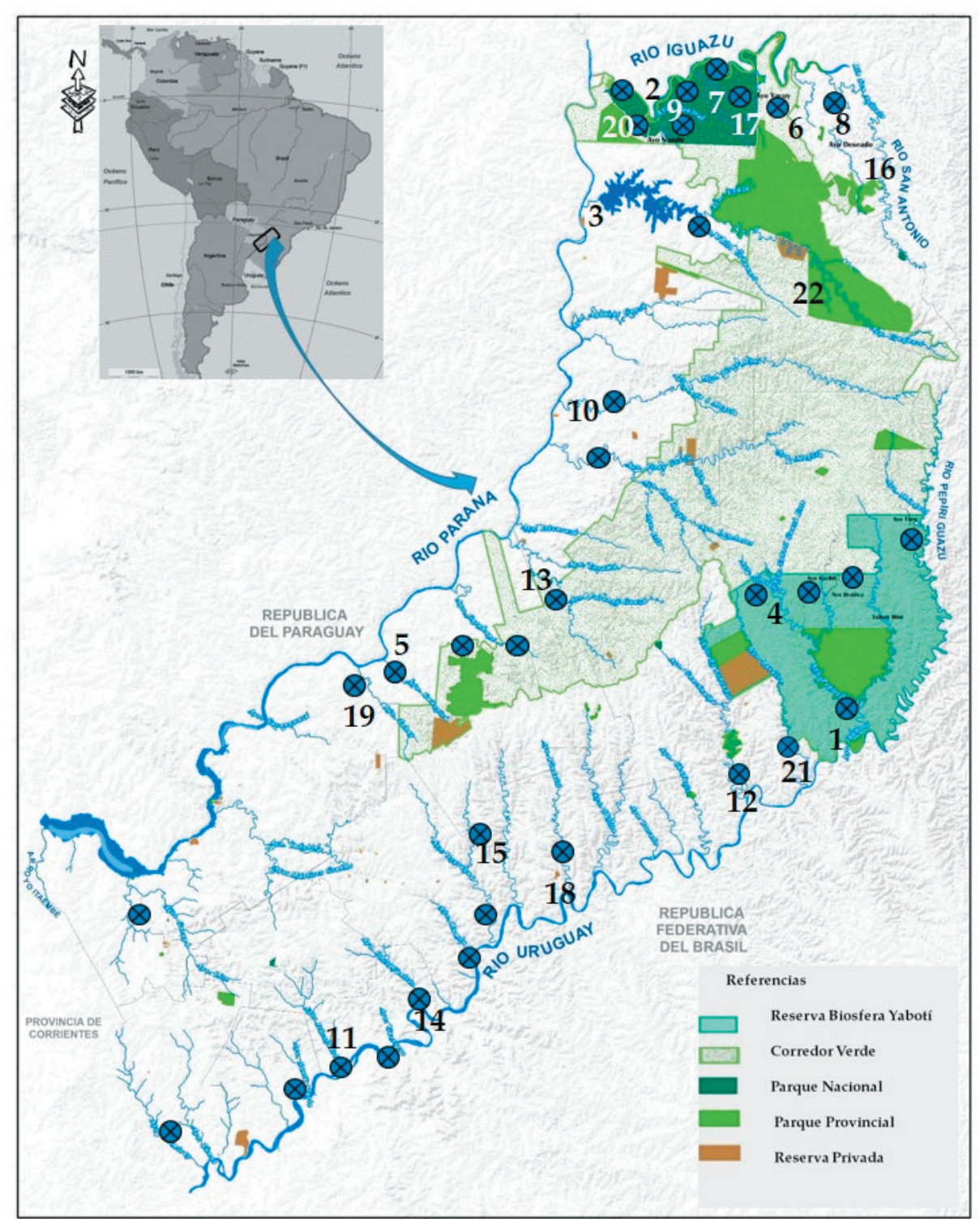

Figura 1. Ubicación de los arroyos con peces de distribución endémica en la provincia de Misiones, Argentina (indicados con un círculo azul). El número junto a cada arroyo señala el orden de prioridad de conservación asignado a cada uno (Tabla 3).

Figure 1. Location of the streams with endemic species of fish in the province of Misiones, Argentina (indicated with blue circles). Numbers indicate the order of priority of conservation of the streams (Table 3).

representar a todas las especies (Vane-Wright et al. 1991; Romdhane et al. 2018). En el caso que dos arroyos pudiesen contribuir con las mismas especies, se priorizó aquel con mayor cantidad de especies totales.

\section{Resultados}

Se registraron 79 especies de peces endémicos de la provincia de Misiones, clasificadas dentro de 4 órdenes y 12 familias (Tabla 1), valor que representa el $14 \%$ de las especies de peces de agua dulce de la Argentina (570 especies)
(Mirande and Koerber 2020). De ese total, 39 especies son endémicas exclusivamente de los arroyos de Misiones y otras 40 también están presentes en cursos de agua del sudeste de Brasil, del norte de Uruguay y del este de Paraguay (Tabla 1). Estos taxones se distribuyen en 36 arroyos (Figura 1), ocupando - con riquezas similares las tres cuencas - tributarios al río Iguazú (T.IGUZ, 31 especies), al río Paraná (T.PAR, 28 especies) y al río Uruguay (T.URU, 29 especies). Se destacan ciertas particularidades en la distribución: 1) sólo una especie (Australoheros angiru) es 
Tabla 1. Especies de peces endémicas de la Selva Atlántica presentes en arroyos y ríos de las tres cuencas tributarias de la provincia de Misiones, Argentina. Algunas de las especies son exclusivas de Misiones (39 especies), mientras otras (40 especies) también aparecen en sitios cercanos de Brasil (BR), Paraguay (PR) y Uruguay (UR). Con el número 1 se indica la presencia de la especie en cada una de las tres cuencas tributarias: T.IGUZ (río Iguazú y tributarios), T.PAR (tributarios al río Paraná) y T.URU (tributarios al río Uruguay).

Table 1. Species of endemic fish of the Atlantic Forest in streams and rivers of the three main basins in the province of Misiones, Argentina. Some species are restricted to Misiones (39 species), while others (40 species) also inhabit close watercourses in Brazil (BR), Paraguay (PR) and Uruguay (UR). A number 1 indicates the presence of a species in each of the main three basins: T.IGUZ (Iguazú river and tributaries), T.PAR (tributaries to the Paraná river) and T.URU (tributaries to the Uruguay river).

T.IGUZ T.PAR T.URU ARROYO-CUENCA

\begin{tabular}{|c|c|c|c|c|c|}
\hline & & T.IGUZ & T.PAR & T.URU & ARROYO-CUENCA \\
\hline & CHARACIFORMES & & & & \\
\hline & ANOSTOMIDAE & & & & \\
\hline 1 & $\begin{array}{l}\text { Leporinus amae } \\
\text { CHARACIDAE }\end{array}$ & & & 1 & Fortaleza, Toro, Yabotí Miní, Paraíso (BR) \\
\hline 2 & Diapoma obi & & 1 & & Paranay-Guazú \\
\hline 3 & Diapoma lepiclastus & & & 1 & Fortaleza, Ramos (BR) \\
\hline 4 & Diapoma uruguayensis & & & 1 & Fortaleza, Ramos y Santa María (UR) \\
\hline 5 & Diapoma nandi & & 1 & & Piray Miní \\
\hline 6 & Hypobrycon poi & & & 1 & Once vueltas, Acaraguá (BR, UR) \\
\hline 7 & Psalidodon bifasciatus & 1 & & & Ñandú, Arrechea, Yacuí (BR) \\
\hline 8 & Astyanax dissimilis & 1 & & & Ñandú, Apepú, Arrechea (BR) \\
\hline 9 & Psalidodon ita & 1 & & & Tateto-San Antonio- Yacuí \\
\hline 10 & Psalidodon leonidas & & 1 & & Urugua-í \\
\hline 11 & Psalidodon ojiara & & & 1 & Benitez-Yabotí \\
\hline 12 & Andromakhe paris & & & 1 & Yabotí, Fortaleza \\
\hline 13 & Andromakhe saguazu & & & 1 & Shangai, Once Vueltas, Yabotí, Ramos (BR, UR) \\
\hline 14 & Psalidodon troya & & 1 & & Cuñapirú, Tabay, \\
\hline 15 & Andromakhe tupi & & 1 & & Cuñapirú \\
\hline 16 & Bryconamericus agna & & 1 & & Tabay-Cuñapirú, Garuhape \\
\hline 17 & Bryconamericus ikaa & 1 & & & Tateto-San Antonio, Verde, Deseado \\
\hline 18 & Bryconamericus mennii & & 1 & & Cuñapirú, Urugua-í \\
\hline 19 & Bryconamericus pyahu & 1 & & & Tateto-San Antonio, Yacuí \\
\hline 20 & Bryconamericus sylvicola & & 1 & & Central-Urugua-í \\
\hline 21 & Bryconamericus uporas & & & 1 & Yabotí, Fortaleza-Yabotí, Once Vueltas, Toro \\
\hline 22 & Bryconamericus ytu & & & 1 & Shangai \\
\hline 23 & Oligosarcus amome & & & 1 & Oveja Negra y Fortaleza-Yabotí \\
\hline 24 & $\begin{array}{l}\text { Oligosarcus menezesi } \\
\text { ERYTHRINIDAE }\end{array}$ & 1 & 1 & & Urugua-í, 42 Pozón (Salto Dos Hermanas) Iguazú \\
\hline 25 & $\begin{array}{l}\text { Hoplias australis } \\
\text { PARODONTIDAE }\end{array}$ & & & 1 & Itacaruaré, Santa María (BR, UR) \\
\hline 26 & $\begin{array}{l}\text { Apareiodon vittatus } \\
\text { SILURIFORMES } \\
\text { PIMELODIDAE }\end{array}$ & 1 & & & Yacuí (BR) \\
\hline 27 & Pimelodus britskii & 1 & & & Verde o Deseado, Iguazú (BR) \\
\hline 28 & Pimelodus ortmanni & 1 & & & Iguazú (BR) \\
\hline 29 & $\begin{array}{l}\text { Steindachneridion melanodermatum } \\
\text { AUCHENIPTERIDAE }\end{array}$ & 1 & & & Iguazú (BR) \\
\hline 30 & Glanidium ribeiroi & 1 & 1 & & Iguazú, Ñandú, Los Dos Hermanos, Deseado, \\
\hline 31 & $\begin{array}{l}\text { Tatia jaracatia } \\
\text { HEPTAPTERIDAE }\end{array}$ & 1 & & & $\begin{array}{l}\text { Urugua-í, Cuñapirú (BR) } \\
\text { Deseado(BR) }\end{array}$ \\
\hline 32 & Heptapterus mbya & & 1 & & Cuñapirú, Azul-Moreno-Garuhapé \\
\hline 33 & Heptapterus mandimbusu & & & 1 & Melo \\
\hline 34 & Imparfinis hollandi & 1 & 1 & & Ibicuy, Urugua-í \\
\hline 35 & Rhamdia branneri & 1 & & & Arroyo sin nombre PNI (BR) \\
\hline 36 & Rhamdia voulezi & 1 & & & Apepú, Dos Hermanas PNI (BR) \\
\hline 37 & $\begin{array}{l}\text { Rhamdella cainguae } \\
\text { LORICARIIDAE }\end{array}$ & & 1 & & Cuña Pirú, Urugua-í, Tabay, Horqueta \\
\hline 38 & Ancistrus mullerae & 1 & & & Dos Hermanas, Santo Domingo (BR) \\
\hline 39 & Eurycheilichthys pantherinus & & & 1 & Garibaldi-Yabotí (BR) \\
\hline 40 & Hypostomus derbyi & 1 & 1 & & Iguazú, Urugua-í, Uruzú (BR) \\
\hline 41 & Hypostomus myersi & 1 & 1 & & Iguazú, Urugua-í (BR) \\
\hline 42 & Hisonotus aky & & & 1 & Garibaldi, Fortaleza-Yabotí \\
\hline 43 & Hisonotus yasi & 1 & & & Lobo-Iguazú \\
\hline 44 & Hisonotus hungy & & 1 & & Arroyo Tirica-Urugua-í \\
\hline 45 & Rineloricaria misionera & & 1 & 1 & Pindapoy, Liso, Santa Ana, Cuñapirú, Tateto, \\
\hline 46 & Rineloricaria reisi & & & 1 & $\begin{array}{l}\text { Pepirí Miní } \\
\text { Paraiso (BR) }\end{array}$ \\
\hline 47 & Rineloricaria stellata & & & 1 & Yabotí Miní, San Juan-Yabotí (BR) \\
\hline 48 & $\begin{array}{l}\text { Rineloricaria zaina } \\
\text { CALLICHTHYDAE }\end{array}$ & & & 1 & Yabotí Miní-Yabotí (BR) \\
\hline 49 & Corydoras carlae & 1 & 1 & & Iguazú, Urugua-í (BR) \\
\hline
\end{tabular}


Tabla 1. Continuación.

Table 1. Continuation TRICHOMYCTERIDAE

51 Cambeva stawiarski

52 Cambeva ytororo

53 Scleronema milonga CYPRINODONTIFORMES

POECILIIDAE

54 Cnesterodon pirai

55 Phalloceros harpagos

PERCIFORMES

CICHLIDAE

56 Australoheros tembe

57 Australoheros ykeregua

58 Australoheros angiru

59 Australoheros kaaygua

60 Crenicichla hu

61 Crenicichla yjhui

62 Crenicichla tesay

63 Crenicichla yaha

64 Crenicichla ypo

65 Crenicichla missioneira

66 Crenicichla gaucho

67 Crenicichla minuano

68 Crenicichla tendybaguassu

69 Crenicichla celidochilus

70 Crenicichla hadrostigma

71 Crenicichla iguassuensis

72 Crenicichla mandelburgeri

73 Crenicichla tapii

74 Crenicichla tuca

75 Gymnogeophagus che

76 Gymnogeophagus costellatus

77 Gymnogeophagus jaryi

78 Gymnogeophagus lipokarenos

79 Gymnogeophagus taroba

1

1

1

1

1

1

1

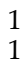

compartida entre T.URU y T.IGUZ, 2) siete especies comparten T.IGUZ y T.PAR, y 3) 13 especies se registraron en un solo curso de agua (Tabla 1 ).

El valor de QBR de los arroyos varió entre 40 y 100 (ver Material Suplementario). Los arroyos de las cuencas T.IGUZ y T.URU registraron valores máximos de QBR (Figuras $2 \mathrm{~A}$ y $2 \mathrm{~B}$, respectivamente). Los arroyos T.PAR alcanzaron un valor máximo de 80 (Figura 2C). En las tres cuencas se registraron arroyos con algún grado de alteraciones ecológicas;
Yacuí (BR)

Yacuí, Arroyos Sendero Yacaratía viejo (BR)

Saltos del Tabay

1 Itacaruare, IJui y Santa Rosa (BR)

Arroyo Almeida-Cuñapirú

Yacaratiá, Arrechea, arroyo A2 Puerto Macuco (PR, BR)

Urugua-í

1 Fortaleza, Paraíso, Shangai, Guerrero, Tamandúa

1 Soberbio, Iguazú (BR)

Lobo, Nandú, Yacui

Piray Miní

Urugua-í

Verde-Iguazú (BR)

Urugua-í

Urugua-1

1 Fortaleza, Yabotí, Paraíso (BR)

1 Fortaleza, Soberbio (BR)

1 Fortaleza, Soberbio, Yabotí (BR)

1 Fortaleza, Soberbio, Paraíso (BR)

1 Soberbio, Yabotí, Paraíso (UR)

1 Yabotí, río Uruguay (BR)

s/n arroyo dentro PNI (BR)

Cuñapirú, Garuhape, Piray Mini Iguazú (PR)

Iguazú, Ñandú (BR)

Iguazú, Deseado, Ñandú (BR)

Tirica, Urugua-í

1 Itacaruaré, Chimiray Miní

Cuña Pirú, Nacanguazu (PR)

1 Paraíso, Fortaleza, Melo, Guerrero, Shangai (BR) Ñandú, Deseado, Iguazú

el menor valor QBR (40) se presentó en la cuenca T. IGUZ (Figura 2A). Más de la mitad de los cursos de agua interiores se encuentran incluidos en la categoría 'no protegido' (Tabla 2).

Sobre la base de los resultados de la distribución de las 79 especies en los 36 cursos de agua se determinaron los arroyos prioritarios para su conservación (Tabla 3), ordenados de manera decreciente según la cantidad de especies que aportan. La conservación adecuada de 22 cursos fluviales

Tabla 2. Grado de protección de arroyos con peces de distribución endémica en la provincia de Misiones, Argentina. T.IGUZ: río Iguazú y tributarios; T.PAR: tributarios al río Paraná; T.URU: tributarios al río Uruguay.

Table 2. Degree of protection of streams with endemic fish species in the province of Misiones, Argentina. T.IGUZ: Iguazú river and tributaries; T.PAR: tributaries to the Paraná river; T.URU: tributaries to the Uruguay river.

\begin{tabular}{lcccc}
\hline Categoría de protección & T.IGUZ & T.PAR & T.URU & Total \\
\hline No protegido (0\%) & - & 8 & 10 & 18 \\
Escasamente protegido (0.01\%-19.99\%) & 2 & & - & 2 \\
Relativamente protegido (20-49.99\%) & - & - & 1 & 1 \\
Moderadamente protegido (50-79.99\%) & 2 & 2 & 2 & 6 \\
Bien protegido (80-100\%) & 6 & - & 3 & 9 \\
Total arroyos & 10 & 10 & 16 & 36 \\
\hline
\end{tabular}




\section{A Verde-Deseado Tateto-San Antonio \\ Iguazu \\ Lobos_Iguazú Ibicuy \\ Dos Hermanas \\ \begin{tabular}{r|} 
Yacuy \\
arroyos S/N, PNI \\
Ñandu \\
Apepú, Arrechea
\end{tabular}}

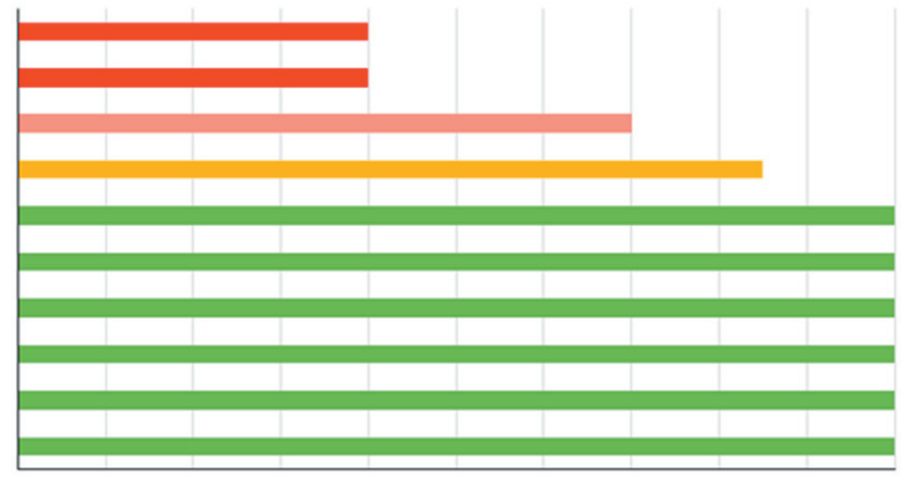

0
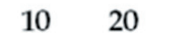

30

$40 \quad 50$

60

$80 \quad 90 \quad 100$

QBR

B Chimiray Chico

Pindapoy-Santa Ana

Paranaí Guazú

Tabay

Garuhape

Piray Miní

Urugua-í

Tateto

Cuña Pirú

Tirica

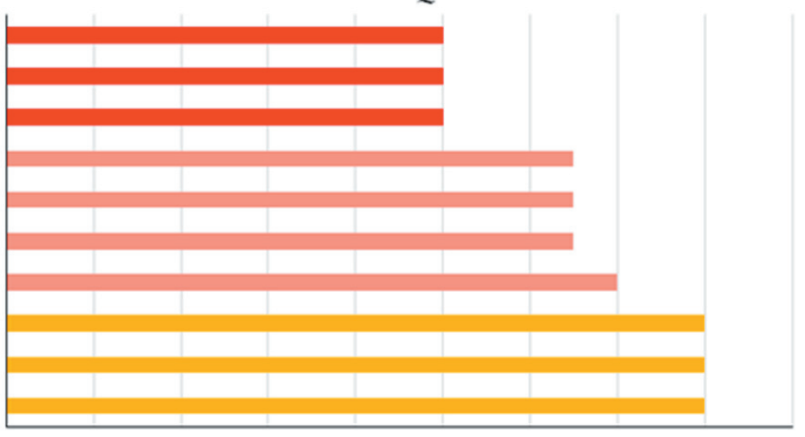

10

$20 \quad 30$

$40 \quad 50$

C

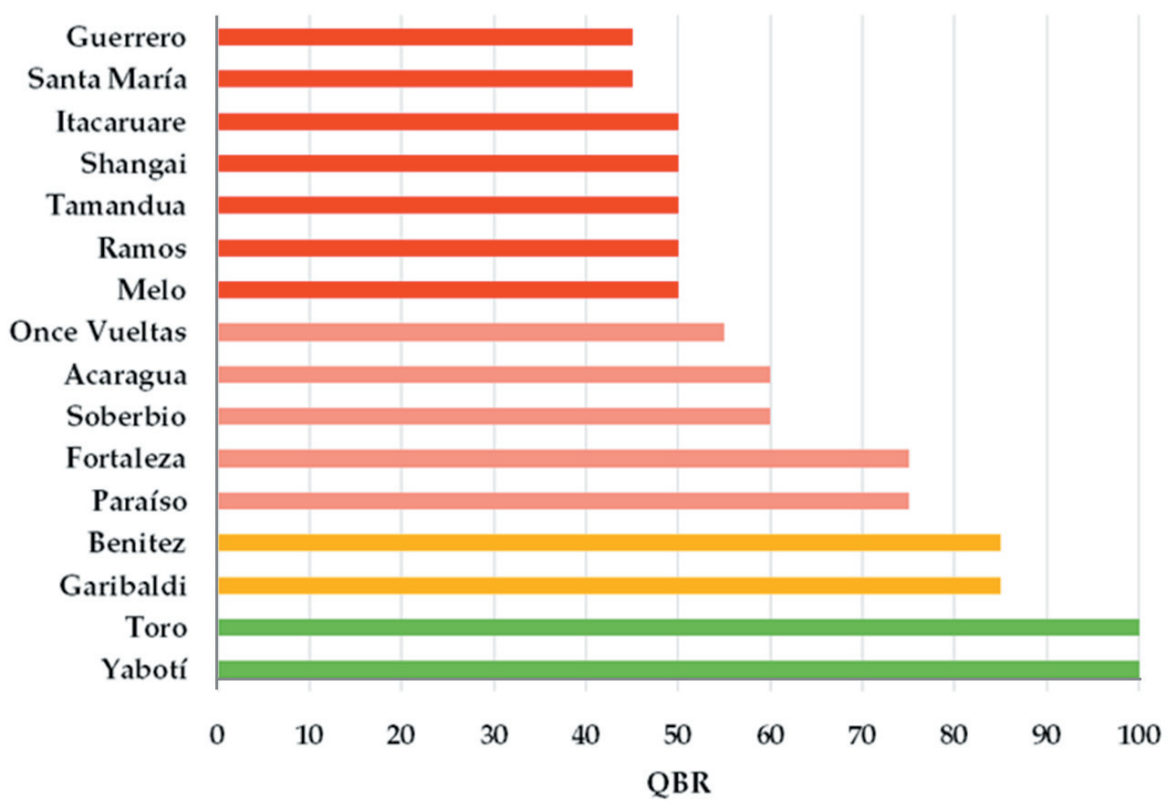

Figura 2. Índice de calidad ecológica (QBR) de arroyos con peces de distribución endémica en la provincia de Misiones, Argentina. Categorización según Munné et al. (1998): verde (muy buena), amarillo (buena), rosa (intermedia), rojo (alteración fuerte) y negro (degradación extrema). A: río Iguazú y sus tributarios (T.IGUZ); B: tributarios del río Paraná (T.PAR); C: tributarios del río Uruguay (T.URU).

Figure 2. Ecological quality index (QBR) of streams with fish of endemic distribution in the province of Misiones, Argentina. Classification according to Munné et al. (1998): green (very good), yellow (good), pink (intermediate), red (strong alteration) and black (extreme degradation). A: Iguazú river and tributaries (T.IGUZ); B: Paraná river tributaries (T.PAR); C: Uruguay river tributaries (T.URU). 
(2.5\% del total de los cursos de agua en Misiones) permitiría proteger todas las especies conocidas de peces que, dentro de la Argentina, están presentes sólo en la provincia de Misiones. En la actualidad, sólo cinco de esos cursos de agua poseen una protección adecuada (el arroyo Yabotí Miní y los arroyos que transcurren dentro del Parque Nacional Iguazú); otros siete arroyos poseen una protección intermedia y los siete restantes tienen protección nula o insuficiente (Tabla 3).

Tabla 3. Arroyos, en orden de prioridad (OP), para conservar la totalidad de peces con distribución endémica de Misiones, Argentina, especificando la cantidad de especies en cada uno (y acumuladas según el OP), sus categorías de estado ecológico general (EG; MB: muy bueno; B: bueno; IN: intermedio; AF: alteración fuerte), su grado de protección (GP; BP: bien protegido; MP: moderadamente protegido; EP: escasamente protegido; NP: no protegido) y las amenazas y necesidades de conservación en sus cuencas. PNI: Parque Nacional Iguazú.

Table 3. Streams, in order of priority (OP), to protect all species of endemic fish in the province of Misiones, Argentina, detailing the number of fish species in each one (and accumulated according to their OP), their general ecological status (EG; MB: very good; B: good; IN: intermediate; AF: strong alteration), their degree of protection (GP; BP: well protected; MP: moderately protected; EP: poorly protected; NP: not protected) and the main threats and needs for their conservation. PNI: Iguazú National Park.

\begin{tabular}{|c|c|c|c|c|c|c|}
\hline $\mathrm{OP}$ & Arroyo/río & $\begin{array}{l}\text { Especies } \\
\text { propias }\end{array}$ & $\begin{array}{l}\text { Especies } \\
\text { acumuladas }\end{array}$ & EG & GP & Amenazas / Necesidades \\
\hline 1 & Yabotí & 16 & 16 & MB & $\mathrm{BP}$ & $\begin{array}{l}\text { Cabecera desprotegida y pesca furtiva en todo } \\
\text { el cauce }\end{array}$ \\
\hline 2 & Iguazú & 13 & 29 & IN & MP & $\begin{array}{l}\text { Margen argentina desprotegida en parte y } \\
\text { peces exóticos }\end{array}$ \\
\hline 3 & Urugua-í & 9 & 38 & IN & MP & $\begin{array}{l}\text { Cabecera desprotegida en parte, represa } \\
\text { hidroeléctrica, peces exóticos }\end{array}$ \\
\hline 4 & Fortaleza & 6 & 44 & B & MP & $\begin{array}{l}\text { Cuenca alta desprotegida y altamente } \\
\text { antropizado }\end{array}$ \\
\hline 5 & Cuña Pirú & 6 & 50 & IN & MP & $\begin{array}{l}\text { Cuenca baja y media y nacientes sin } \\
\text { protección, zona de monocultivos y ganadería }\end{array}$ \\
\hline 6 & Yacuy & 6 & 56 & IN & MP & $\begin{array}{l}\text { Límite PNI: alto riesgo de caza y pesca } \\
\text { furtiva. } \\
\text { Deforestación margen este }\end{array}$ \\
\hline 7 & Apepú, Arrechea (Iguazú) & 3 & 59 & MB & $\mathrm{BP}$ & No presenta \\
\hline 8 & Verde Deseado & 3 & 62 & IN & EP & Cuenca alta en parte protegida \\
\hline 9 & Arroyo sin nombre (PNI) & 2 & 64 & MB & $\mathrm{BP}$ & No presenta \\
\hline 10 & Piray Miní & 2 & 66 & IN & NP & Urgente protección y restauración \\
\hline 11 & Itacaruare & 2 & 68 & $\mathrm{AF}$ & NP & Urgente protección y restauración \\
\hline 12 & Dos Hermanas & 1 & 69 & MB & $\mathrm{BP}$ & No presenta \\
\hline 13 & Paraíso & 1 & 70 & $\mathrm{~B}$ & MP & $\begin{array}{l}\text { Ausencia control de pesca. Deforestación } \\
\text { margen derecha }\end{array}$ \\
\hline 14 & Paraná y Guazú & 1 & 71 & $\mathrm{AF}$ & NP & Urgente protección y restauración \\
\hline 15 & Once Vueltas & 1 & 72 & IN & NP & Urgente protección y restauración \\
\hline 16 & Tateto-San Antonio & 1 & 73 & IN & NP & Urgente protección y restauración \\
\hline 17 & Tirica & 1 & 74 & IN & MP & Urgente protección y restauración \\
\hline 18 & Soberbio & 1 & 75 & IN & $\mathrm{RP}$ & Urgente protección y restauración \\
\hline 19 & Melo & 1 & 76 & $\mathrm{AF}$ & NP & Urgente protección y restauración \\
\hline 20 & Ibicuy & 1 & 77 & MB & $\mathrm{BP}$ & No presenta \\
\hline 21 & Shangai & 1 & 78 & $\mathrm{AF}$ & NP & Urgente protección y restauración \\
\hline 22 & Tabay & 1 & 79 & IN & NP & Urgente protección y restauración \\
\hline
\end{tabular}




\section{Discusión}

La región de los arroyos de la provincia de Misiones tiene características hidrogeológicas y geomorfológicas que permiten la existencia de una ictiofauna diferenciada, con alto grado de endemismos (Bonetto 1998; López et al. 2002, 2008; Menni 2004; López and Miquelarena 2005). Esto se atribuye al aislamiento que genera la presencia de saltos y cascadas, que representan una barrera natural para la distribución de las especies. Estudios del género Crenicichla apoyan esta hipótesis al mostrar que los saltos del Moconá, las cataratas del Iguazú y los saltos del Guairá produjeron la diferenciación de varios de sus clados (Piálek et al. 2011, 2015). Por otra parte, Ríčan et al. (2011) establecen que los patrones de distribución de las especies de Australoheros en las cuencas de los ríos Uruguay e Iguazú señalan una conexión histórica de cuencas que no se mantiene en la actualidad (río Iguazú inferior con el arroyo Urugua-í y el río Iguazú medio con el Urugua-í en su tramo superior). Como resultado de estos procesos, el río Iguazú y sus tributarios contienen el mayor número de peces endémicos (31 especies) del territorio misionero y una de las mayores prioridades de conservación, en coincidencia con estudios que proponen que $70 \%$ de la ictiofauna del río Iguazú es endémica (Garavello et al. 1997; Albert and Reis 2011; Baumgartner et al. 2012; Casciotta et al. 2016). Otros dos arroyos tributarios del río Uruguay (Yabotí y Fortaleza) son los que tienen más especies endémicas (16 y 13 especies, respectivamente), por lo que, junto con los arroyos Benítez y Garibaldi, se propone que sean considerados una unidad de gestión para su conservación. Un sector de esta cuenca forma parte de la Reserva de Biósfera Yabotí, con una alta diversidad íctica y ambientes que aún conservan una integridad ecológica elevada (Araya et al. 2012; Flores et al. 2015, 2020). Otro arroyo prioritario es el Urugua-í (T.PAR), que es el curso de agua interior más extenso $(246 \mathrm{~km})$ de la provincia de Misiones y el tercero en número de especies endémicas (12 especies), lo que justifica su alta prioridad.

Los sistemas lóticos son subsidiados por material alóctono para el mantenimiento de las comunidades bióticas y la alimentación de los peces, dependiente de la vegetación marginal (Lowe-McConnell 1999). La vegetación riparia es un elemento estructural importante de los ecosistemas acuáticos por su aporte en alimentos, refugio para la reproducción y otros factores reguladores de la comunidad íctica (Sabino and Zuanon 1998; Araya et al. 2012). Los insectos acuáticos o terrestres en distintas etapas de desarrollo juegan un rol significativo en la alimentación de los peces, así como la materia orgánica y los detritos por su contenido en microorganismos (Flores et al. 2009; Hirt et al. 2011). En consecuencia, eliminar la vegetación marginal resulta en pérdida de biodiversidad, reducción de la calidad del agua y degradación de las cuencas hidrográficas (Harrison 2002). Por eso, los arroyos de menor calidad ecológica se ubican en zonas muy productivas de Misiones (e.g., T.IGUZ: Tateto, San Antonio; T.PAR: Paranaí y Pindapoy; T.URU: Santa María, Guerrero, Itacaruare), en gran medida por el mal estado de conservación de la vegetación marginal.

El uso y el manejo del suelo asociados a la contaminación de las cuencas hidrográficas es una problemática mundial (Ongley 1997; Harrison 2002). En las áreas más productivas y modificadas de la Argentina, como la región Pampeana, la baja calidad de ribera está asociada a tramos urbanos (Bertora et al. 2018) y a regiones de ganadería irrestricta (Feijoó et al. 2012), con repercusiones en la estructura de la comunidad de peces (Granito et al. 2016). La degradación de los sistemas fluviales de Misiones está asociada especialmente a las actividades agrícolas en las cuencas (Placci and Di Bitetti 2005; CIC-OEA 2017). Las principales actividades económicas que llevaron a la reconversión del bosque nativo incluyen cultivos anuales (i.e., caña de azúcar, maíz, tabaco) y cultivos perennes de especies exóticas (i.e., pino, eucaliptos, paraísos) que afectan el crecimiento de las autóctonas y la formación de sotobosques (Laclau 1994; Drozd et al. 2010). En los últimos 20 años, la cría de ganado es una actividad económica relevante, en particular en el sur de la provincia. En este trabajo encontramos que los cursos de agua con alteraciones ecológicas fuertes e intermedias se presentan en los tributarios del río Paraná (T.PAR), la zona de mayor desarrollo forestal de Misiones, así como en la zona centro-norte de la provincia (T.URU), donde se concentra el cultivo de tabaco (Ministerio de Hacienda de la Nación 2018). $\mathrm{Al}$ analizar la reconversión forestal en la zona centro de la provincia Misiones, Drozd et al. (2010) destacan que en las áreas protegidas del Valle del Cuña Pirú y sus alrededores persistió un bosque relativamente continuo que cubre $\sim 500 \mathrm{~km}^{2}$. A su vez, cerca de $50 \%$ del bosque permanente se presentó en 
parches con superficies menores a 100 ha. Dichos fragmentos estuvieron levemente asociados a cursos de agua. Es probable que esto haya obedecido al cumplimiento de la Ley de Bosques Protectores, que protege a todas aquellas formaciones boscosas que se encuentran en una faja correspondiente a tres veces el ancho del cauce y no menor a $5 \mathrm{~m}$ a cada orilla de los arroyos (Ley Prov. 3426).

Las modificaciones del flujo de agua de un río o arroyo provocados por represas altera de forma severa su hidrología, con modificaciones ambientales y formación de nuevos hábitats que pueden colonizar especies generalistas, más flexibles en cuanto a necesidades alimentarias y de reproducción (Agostinho et al. 2007). Estos aspectos son claves en ríos de bajo orden con faunas adaptadas a ambientes lóticos, lo cual puede ser decisivo en la pérdida de biodiversidad. Tal es el caso de Crenicichla yaha, que fue colectada en 1986 durante los estudios de impacto ambiental previos a la construcción de la presa hidroeléctrica Urugua-í, una obra que en 1990 interrumpió la conexión con el río Paraná, inundando su cuenca baja y media y provocando cambios notables en su ictiofauna. Si bien la especie se creía extinta (Piálek et al. 2015), se volvió a reportar en 2016 aguas arriba del embalse (Ř́ćan et al. 2017). Esos cambios suelen estar asociados a la introducción de especies exóticas, como ocurrió con Tilapia cf. rendalli en Urugua-í (Iwaszkiw 2006). La introducción de especies exóticas constituye una amenaza para la fauna autóctona por competencia por el recurso alimentario, por depredación excesiva o por alteraciones genéticas (Agostinho et al. 2007). En Misiones, la principal vía de introducción son los escapes o liberaciones provenientes de establecimientos o producción piscícola (Meichtry de Zaburlin et al. 2013). Las políticas gubernamentales, junto con inversiones privadas, han permitido el crecimiento de la piscicultura en la provincia, con especies oportunistas que pueden establecerse, incluso, en ambientes degradados, como Cyprinus carpio, Oreochromis niloticus y Tilapia sp., citadas en Misiones (López et al. 2005). En los sistemas fluviales vecinos de Brasil hay un número mayor de especies exóticas, entre las cuales se incluyen tilapias (Tilapia rendalli y Oreochromis niloticus), tucunaré (Cichla kelberi), bagre americano (Ictalurus punctatus), carpa plateada (Hypoptalmichthys molotrix), blackbass (Micropterus salmoides) y bagre africano (Clarias gariepinus), este último de presencia comprobada en el río Iguazú (Baumgartner et al. 2012; Casciota et al. 2016), donde representa una amenaza real para las especies endémicas. En general, el manejo de los recursos acuáticos en Misiones (especialmente de peces) es pobre y no se basa en información técnica ni científica. A lo largo de la historia, las acciones de manejo han incluido sólo el control de pesquerías y del stock pescable por medio del establecimiento de épocas de veda (Araya et al. 2009).

Nuestros resultados muestran que la conservación adecuada de sólo 22 de los arroyos misioneros (2.5\% del total) permitiría proteger todas las especies conocidas de peces con distribución exclusiva en la provincia de Misiones. Estos arroyos tienen escaso grado de protección en la actualidad, más de la mitad están desprotegidos y únicamente $15 \%$ está contenidodentro deun Área NaturalProtegida. Aun cuando la provincia de Misiones posee un sistema de áreas protegidas extenso en el ámbito nacional (15.3\% de su superficie) (Burkart et al. 2007), su diseño responde mayormente a aspectos de conservación de ecosistemas terrestres y presenta deficiencias en relación con los sistemas acuáticos (Giraudo 2017; SiFAP 2019). La mayoría de las áreas protegidas en la Argentina se diseñaron sin contemplar aspectos de la biodiversidad de sistemas acuáticos $\mathrm{y}$, por lo tanto, resultan deficientes para proteger los cuerpos de agua y su biodiversidad asociada (Boscarol 2013). Una situación similar se presenta en los sistemas fluviales de Brasil (Agostinho et al. 2005; Belei et al. 2016). Si bien hay tres reservas naturales ícticas en Misiones (sobre el Paraná: Caraguatay [81 ha] y Corpus [882 ha]; sobre el Uruguay: Monumento Natural Nacional Saltos del Moconá) (Giraudo et al. 2003a), nuestros resultados dan cuenta de que los arroyos con especies endémicas están moderadamente protegidos (i.e., Urugua-í, Yacuy, Cuña Pirú, Fortaleza) o carecen de protección (i.e., Piraí Guazú y Tateto, entre otros). Giraudo et al. (2003b) mostraron que los ríos Uruguay y el Alto Paraná fueron olvidados en las estrategias de conservación de la Selva Atlántica, cuyas selvas en galería tienen un estado de conservación bastante crítico que necesita de acciones urgentes como la creación de áreas protegidas, la legislación y el control sobre la conservación de los bosques protectores. A pesar de que la provincia cuenta con leyes de protección ambiental (Ley XVI $N^{\circ} 8$ [antes 1040]: Régimen de Protección de la Fauna Íctica; Ley 3426: Ley de Bosques 
Protectores), no son suficientes para proteger de manera adecuada los cursos fluviales provinciales y su ictiofauna endémica. Por caso, una de las áreas protegidas más emblemáticas y extensa de la provincia de Misiones es la Reserva de Biósfera Yabotí (236313 ha), que carece de información sobre el estado de conservación de sus cursos de agua (Ministerio de Ecología y Recursos Naturales Renovables 2011). Problemas como la escasa investigación y fiscalización de las poblaciones de peces, en particular de aquellos usados en la pesca furtiva, la desprotección de subcuencas y amenazas como la modificación de los cursos de agua y su entorno (i.e., bosques protectores hasta los límites de la reserva, grandes obras de infraestructura como represas y puentes) o la contaminación (i.e., desechos de obrajes, turismo, residuos, agroquímicos) dejan en evidencian la necesidad de que el Consejo de Administración de la Reserva de Biósfera Yabotí contemple específicamente a las cuencas hídricas para su gestión y legislación. Esta situación es compartida por la mayoría de los sistemas fluviales de Misiones.

En resumen, mediante este análisis basado en la distribución de peces endémicos y en el estado de conservación del sistema hidrográfico de la provincia de Misiones proponemos un conjunto de 22 arroyos prioritarios para la conservación de las 79 especies de peces endémicos de la Selva
Atlántica en Misiones. En la actualidad, más de la mitad de estos arroyos no poseen protección y muchos sufren alteraciones como destrucción de la vegetación marginal, represamientos, polución e introducción de especies exóticas. Nuestros resultados muestran las deficiencias de los sistemas actuales de áreas protegidas de Misiones en representar a sus ecosistemas acuáticos como consecuencia de la débil o inexistente consideración de estos ecosistemas en la toma de decisiones y diseño de áreas de conservación. Finalmente, ilustramos la aplicación de un protocolo de fácil comprensión y sencilla implementación que permitiría a los gestores evaluar el estado y la prioridad de conservación de los cursos fluviales, y su representatividad en los sistemas de áreas protegidas, detectando vacíos de conservación y maximizando la representación de las especies focales en el menor número posible de cursos protegidos.

Agradecimientos. Queremos agradecer al Dr. Jorge Casciotta, la Dra. Adriana Almirón y la Dra. Mercedes Azpelicueta por la colaboración en la revisión taxonómica de la lista de especies. A los compañeros del Proyecto de Investigación de la Ictiofauna de la Provincia de Misiones FCEQyN-UNaM por el trabajo compartido. Al CONICET que brinda la infraestructura de investigación de uno de los autores (ARG). A los revisores y editores por aportar al mejoramiento del manuscrito.

\section{REFERENCIAS}

Abell, R. 2002. Conservation biology for the biodiversity crisis: A freshwater follow-up. Conservation Biology 16:14351437. https://doi.org/10.1046/j.1523-1739.2002.01532.x.

Agostinho, A. A., S. Thomaz, and L. Gomes. 2005. Conservation of the biodiversity of Brazil's inland waters. Conservation Biology 19:646-652. https://doi.org/10.1111/j.1523-1739.2005.00701.x.

Agostinho, A. A., L. Gomes, and F. Pelecice. 2007. Ecologia e manejo de recursos pesqueiros em reservatórios do Brasil. Eduem Maringa Brasil.

Aguilera, G., M. Benítez, G. Terán G., F. Alonso, and J. Mirande. 2017. A new species of Heptapterus Bleeker 1858 (Siluriformes, Heptapteridae) from the Uruguay River Basin in Misiones, Northeastern Argentina. Zootaxa 4:572-580. https://doi.org/10.11646/zootaxa.4299.4.7.

Albert, J., and J. Reis. 2011. Historical biogeography of Neotropical freshwater fishes. University of California Press, London, England. https://doi.org/10.1525/9780520948501.

Almirón, A., J. Casciotta, L. Piálek, F. Ruiz Díaz, and O. Říčan. 2014a. First record of Rineloricaria reisi, R. stellata and R. zaina (Siluriformes: Loricariidae) from the Río Uruguay basin in Argentina. Check List 10:1528-1530. https://doi.org/ 10.15560/10.6.1528.

Almirón, A., J. Casciotta, M. Azpelicueta, D. Aichino, M. Benítez, L. Piálek, K. Doubnerová, and O. Říčan. 2014b. First record of the genus Tatia (Siluriformes: Auchenipteridae) in freshwaters of Argentina. Ichthyological Contributions of Peces Criollos 27:1-5.

Alonso, F., G. E. Terán, G. Aguilera, O. Řičan, J. Casciotta, and W. S. Serra. 2019. Description of a new species of the Neotropical cichlid genus Gymnogeophagus Miranda Ribeiro, 1918 (Teleostei: Cichliformes) from the Middle Paraná basin, Misiones, Argentina. PLoS ONE 14:e0210166. https://doi.org/10.1371/journal.pone.0210166.

Araya, P., L. Hirt, and S. Flores. 2009. Algunos aspectos de la pesquería artesanal en el área de influencia del embalse Yacyretá. Alto río Paraná, Misiones, Argentina. Boletim do Instituto de Pesca 35:227-238.

Araya, P., L. Hirt, and S. Flores. 2012. Composición y abundancia de la comunidad íctica de la Reserva Biosfera Yabotí. Arroyo Yabotí. Misiones. Argentina. Revista AquaTIC 36:21-27. 
Araya, P., L. Hirt, and S. Flores. 2013. Humedales de los arroyos de Misiones en relieve escarpado. Pp.137-146 en L. Benzaquén et al. (eds.). Inventario de los humedales de Argentina: sistemas de paisajes de humedales del corredor fluvial Paraná Paraguay. Proyecto GEF 4206 PNUD ARG 10/003. Secretaría de Ambiente y Desarrollo Sustentable de la Nación. Buenos Aires, Argentina.

Azpelicueta, M., J. Casciotta, A. Almirón, and S. Körber. 2004. A new species of Otothyrini (Siluriformes: Loricariidae: Hypoptopomatinae) from the Río Uruguay basin, Argentina. Verhandlungen der Gesellschaft für Ichthyologie Band 4:81-90.

Azpelicueta, M., and S. Koerber. 2015. On some freshwater fish species reported by Perugia (1891) from Argentina. Aqua - Journal of Ichthyology and Aquatic Biology 21:39-46.

Baigún, C., N. Oldani, and P. Van Damme. 2011. Represas hidroeléctricas en América Latina y su impacto sobre la ictiofauna. Pp. 395-415 en P. A. Van Damme, F. Carvajal and J. Molina (eds.). Peces de la Amazonía boliviana: potencialidades y amenazas. INIA, Cochabamba, Bolivia.

Baigún, C., D. Colautti, H. López, P. Van Damme, and R. Reis. 2012. Application of extinction risk and conservation criteria for assessing fish species in the lower La Plata River basin, South America. Aquatic Conservation-Marine and Freshwater Ecosystems 22:181-1197. https://doi.org/10.1002/aqc.2223.

Barletta, M., A. Jaureguizar, C. Baigun, N. Fontoura, A. Agostinho, V. Almeida-Val, A. Val, R. Torres, L. Jimenes-Segura, T. Giarrizzo, N. Fabré, V. Batista, and C. Lasso. 2010. Fish and aquatic habitat conservation in South America: a continental overview with emphasis on neotropical systems. Journal of Fish Biology 76:2118-2176. https://doi.org/ 10.1111/j.1095-8649.2010.02684.x.

Baumgartner, G., C. Pavanelli, D. Baumgartner, A. Gasparetto Bifi, T. Debona, and V. Frana. 2012. Peixes do baixo Rio Iguacu. Eduem, Maringá, Brasil. https://doi.org/10.7476/9788576285861.

Belei, F., W. P. Santana Sampaio, P. Giongio, and J. Dergam. 2016. Ictiofauna de área prioritária para conservação, Médio São Francisco, Minas Gerais, sudeste do Brasil. Neotropical Biology and Conservation 11:94-100. https: //doi.org/10.4013/nbc.2016.112.06.

Bertora, A., F. Grosman, P. Sanzano, and J. J. Rosso. 2018. Composición y estructura de los ensambles de peces en un arroyo pampeano con uso de suelo contrastante. Revista del Museo Argentino de Ciencias Naturales nueva serie 20: 11-22. https://doi.org/10.22179/REVMACN.20.545.

Bonetto, A. 1998. Revisión. Panorama sinóptico sobre la ictiofauna, la pesca y piscicultura en los ríos de la cuenca del Plata, con especial referencia al Paraná. Revista de Ictiología 6:3-16.

Boscarol, N. 2013. Áreas protegidas y humedales del Corredor Fluvial Paraná-Paraguay. Pp. 357-373 en L. Benzaquén et al. (eds.). Inventario de los humedales de Argentina: sistemas de paisajes de humedales del corredor fluvial Paraná Paraguay. Proyecto GEF 4206 PNUD ARG 10/003. Secretaría de Ambiente y Desarrollo Sustentable de la Nación. Buenos Aires, Argentina.

Brooks, T., R. Mittermeier, G. da Fonseca, J. Gerlach, M. Hoffmann, J. Lamoreux, C. Mittermeier, J. Pilgrim, and A. Rodrigues. 2006. Global biodiversity conservation priorities. Science 313:58-61. https://doi.org/10.1126/ science.1127609.

Burkart, R., B. Carpinetti, R. Molinari, A. Carminati, G. Martín, A. Balabusic, L. Raffo, N. Machain, M. Almirón, D. Paz Barreto, M. Ochoa, and A. Melhem. 2007. Las áreas protegidas de la Argentina. Herramienta superior para la conservación de nuestro patrimonio natural y cultural. Administración de Parques Nacionales. Buenos Aires. Argentina.

Burlakova, L. E., A. Y. Karatayev, V. A. Karatayev, M. E. May, D. L. Bennett, and M. J. Cook. 2011. Endemic species: contribution to community uniqueness, effect of habitat alteration, and conservation priorities. Biological Conservation 144:155-165. https://doi.org/10.1016/j.biocon.2010.08.010.

Casciotta, J., and A. Almirón. 2008. Crenicichla tesay, a new species of cichlid (Perciformes, Labroidei) from the río Iguazú basin in Argentina. Revue Suisse de Zoologie 115:651-659. https://doi.org/10.5962/bhl.part.80451.

Casciotta, J., A. Almirón, K. Doubnerová, L. Piálek, and O. Říčan. 2015. First records of Characidium heirmostigmata and C. serrano (Characiformes: Crenuchidae) from freshwaters of Argentina. Ichthyological Contributions of Peces Criollos 34:1-3.

Casciotta, J., A. Almirón, L. Ciotek, P. Giorgis, O. Říčan, L. Piálek, K. Dragová, Y. Croci, M. Montes, J. Iwaszkiw, and A. Puentes. 2016. Visibilizando lo invisible. Un relevamiento de la diversidad de peces del Parque Nacional Iguazú, Misiones, Argentina. Historia Natural Tercera Serie 6:5-77.

Casciotta, J., A. Almirón, L. Piálek, and O. Říčan. 2017. Gymnogeophagus taroba (Teleostei: Cichlidae), a new species from the Río Iguazú Basin, Misiones, Argentina. Historia Natural Tercera Serie 7:5-22.

CIC-OEA. 2017. Ecosistemas acuáticos en la Cuenca del Plata. 1a ed. Comité Intergubernamental Coordinador de los Países de la Cuenca del Plata, Ciudad Autónoma de Buenos Aires, Argentina.

Closs, G., M. Krkosek, and J. Olden. 2016. Conservation of freshwater fishes. Cambridge University Press, United Kingdom. https://doi.org/10.1017/CBO9781139627085.

Di Bitetti, M., G. Placci, and L. Dietz. 2003. Una Visión de Biodiversidad para la Ecorregión del Bosque Atlántico del Alto Paraná: Diseño de un Paisaje para la Conservación de la Biodiversidad y prioridades para las acciones de conservación. World Wildlife Fund, Washington, Estados Unidos de Norteamérica.

Di Giacomo, A. S., and O. A. Spitznagel. 2007. Cuenca del río Aguapey. Áreas importantes para la conservación de las aves en Argentina. Sitios prioritarios para la conservación de la biodiversidad. Temas de Naturaleza y Conservación 5:149-151.

Drozd, A., M. Arturi, and S. Torrusio. 2010. Distribución de remanentes (1985-2006) de la Selva Atlántica Interior 
(SAI) en el centro de Misiones, Argentina en función de variables topográficas y edafológicas. RASADEP 1-Número Especial 51:51-66.

Dudley, N. 2008. Directrices para la aplicación de las categorías de gestión de áreas protegidas. Gland, IUCN. Pp. 96. https://doi.org/10.2305/IUCN.CH.2008.PAPS.2.es.

Eken, G., L. Bennun, T. Brooks, W. Darwall, L. Fishpool, M. Foster, and P. Salaman. 2004. Key biodiversity areas as site conservation targets. AIBS Bulletin 54:1110-1118. https://doi.org/10.1641/0006-3568(2004)054[1110: KBAASC]2.0.CO;2.

Feijoó, C., P. Gantes, A. D. Giorgi, J. J. Rosso, and E. Zunino. 2012. Valoración de la calidad de ribera en un arroyo pampeano y su relación con las comunidades de macrófitas y peces. Biología Acuática 27:113-128.

Ferrer, J., and L. Malabarba. 2020. Systematic revision of the Neotropical catfish genus Scleronema (Siluriformes: Trichomycteridae), with descriptions of six new species from Pampa grasslands. Neotropical Ichthyology 18:1-81. https://doi.org/10.1590/1982-0224-2019-0081.

Flores, S., P. Araya, and L. Hirt. 2009. Fish diversity and community structure in a tributary stream of the Paraná River. Acta Limnologica Brasilera 1:57-66.

Flores, S., L. Hirt, and P. Araya. 2015. Estructura y dinámica de la comunidad íctica del arroyo Yabotí, Reserva de Biosfera Yabotí, Misiones, Argentina. Revista Mexicana de Biodiversidad 86:386-395. https://doi.org/10.1016/ j.rmb.2015.04.004.

Flores, S., P. Araya, M. Serrano, G. Barboza, and L. Hirt. 2020. Estructura de la comunidad íctica del arroyo Paraíso afluente del río Uruguay. Misiones. Argentina. Biología Acuática 34:1-12. https://doi.org/10.24215/16684869e009.

Galindo-Leal, C. 2000. Ciencia de la conservación en América Latina. Interciencia 25:129-135.

Garavello, J., C. Pavanelli, and H. Suzuki. 1997. Caracterização da ictiofauna do rio Uguaçu. Pp. 61-84 en A. A. Agostinho and L. A. Gomes (eds.). Reservatório de Segredo. Bases ecológicas para o manejo. Eduem. Maringá, Brasil.

Giraudo, A. 2017. Región humedales misioneros. Pp. 93-110 en L. Benzaquen, R. Blanco, P. Bo, G. Kandus, P. Lingua, P. Minotti and R. Quintana (eds.). Regiones de Humedales de la Argentina. Ministerio de Ambiente y Desarrollo Sustentable. Secretaría de Ambiente y Desarrollo Sustentable de la Nación. Buenos Aires. Argentina.

Giraudo, A., and V. Arzamendia. 2018. Descriptive bioregionalisation and conservation biogeography: what is the true bioregional representativeness of protected areas? Australian Systematic Botany 30:403-413. https://doi.org/ 10.1071/SB16056.

Giraudo, A., E. Krauczuk, V. Arzamendia, and H. Povedano. 2003a. Critical analysis of protected areas in the Atlantic forest of Argentina. Pp. 145-261 en C. Galindo-Leal and I. Câmara (eds.). State of the hotspots: Atlantic forest. Center for Applied Biodiversity Science e Island Press, Washington. USA.

Giraudo, A., H. Povedano, M. Belgrano, U. Pardiñas, A. Miquelarena, D. Ligier, E. Krauczuk, D. Baldo, and M. Castelino. 2003b. Biodiversity of the Argentinian Atlantic Forest. Pp. 160-180 en G. Cámara and C. Galindo-Leal (eds.). State of Hotspots: The Atlantic Forest of South America: Biodiversity Status, Threats and Outlook. Center for Applied Biodiversity Science e Island Press, Washington. USA.

Google. 2020. Mapa hidrográfico de la Provincia Argentina. Google Earth Pro. Versión 7.3.3.7786, Google.

Gómez, S. E., M. González Naya, and L. Ramírez. 2009. Río Iguazú superior: química del agua y comentarios biológicos sobre algunos de sus peces. Pp. 209-222 en B Carpinetti, M. García and M. Almirón (eds.). El Parque Nacional Iguazú. Conservación y desarrollo en la selva paranaense argentina. Administración de Parques Nacionales, Argentina.

Granito, M., J. J. Rosso, M. Boveri, and A. Rennella. 2016. Impacto del uso del suelo sobre la condición de ribera en arroyos pampeanos y su relación con la estructura de la comunidad de peces. Biología acuática 31:19-27.

Harrison, P. 2002. Agricultura mundial: hacia los años 2015/2030. Informe resumido. FAO.

Hirt, L., P. Araya, and S. Flores. 2011. Population structure, reproductive biology and feeding of Astyanax fasciatus (Cuvier, 1819) in an Upper Paraná River tributary, Misiones, Argentina. Acta Limnologica Brasiliensia 23:1-12. https: //doi.org/10.4322/actalb.2011.013.

IPEC. 2015. Gran Atlas de Misiones. Instituto Provincial de Estadística y Censos Gobierno de la Provincia de Misiones. Misiones Argentina.

Iwaszkiw, J. M. 2006. Evaluación de los recursos ícticos del lago Urugua-í (Misiones-Argentina). Consejo Federal de Inversiones. Provincia de Misiones. Misiones Argentina.

Koerber, S., H. Vera-Alcaraz, and R. Reis. 2017. Checklist of the Fishes of Paraguay (CLOFPY). Ichthyological Contributions of Peces Criollos 53:1-99.

Laclau, P. 1994. La conservación de los recursos naturales y el hombre en la Selva Paranaense. Fundación Vida Silvestre Argentina. Boletín Técnico 20. Buenos Aires, Argentina.

Liotta, J. 2016. Base de datos de peces de aguas continentales de Argentina. Publicación Electrónica. URL: pecesargentina.com.ar.

Litz, T., and S. Koerber. 2014. Check List of the Freshwater Fishes of Uruguay (CLOFFUY). Ichthyological Contributions of Peces Criollos 28:1-40.

López, H., C. Morgan, and M. Montenegro. 2002. Ichthyological ecoregions of Argentina. Probiota 1:1-68.

López, H., and A. Miquelarena. 2005. Biogeografía de los peces continentales de la Argentina. Pp. 509-550 en J. Bousquets and J. Morrone (eds.). I Jornadas Biogeográficas de la Red Iberoamericana de Biogeografía y Entomología Sistemática. RIBES XIII-CYTED, Mexico, D.F.

López, H., C. Morgan, and J. Ponte Gómez. 2005. Biodiversidad y Distribución de la Ictiofauna Mesopotámica. INSUGEO 
Miscelánea 14:311-353.

López, H., R. Menni, M. Donato, and A. Miquelarena. 2008. Biogeographical revision of Argentina (Andean and Neotropical Regions): an analysis using freshwater fishes. Journal of Biogeography 35:1564-1579. https://doi.org/ 10.1111/j.1365-2699.2008.01904.x.

Lowe-McConnell, R. 1999. Estudios Ecológicos de Comunidades de Peixes Tropicais. Edusp. São Paulo Brasil.

Margules, C., and R. Pressey. 2000. Systematic conservation planning. Nature 405:243-253. https://doi.org/10.1038/ 35012251.

Meichtry de Zaburlin, N., J. Peso, and P. Araya. 2013. Humedales del Embalse Yacyretá y ambientes asociados. Pp.113122 en L. Benzaquén et al. (eds.). Inventario de los humedales de Argentina: sistemas de paisajes de humedales del corredor fluvial Paraná Paraguay. Proyecto GEF 4206 PNUD ARG 10/003. Secretaría de Ambiente y Desarrollo Sustentable de la Nación. Buenos Aires, Argentina.

Menni, R. C. 2004. Peces y ambientes de la Argentina continental. Monografías Museo Argentino de Ciencias Naturales 5:1-316.

Ministerio de Ecología y Recursos Naturales Renovables. 2019. Diagnóstico de Amenazas a la Biodiversidad de la Reserva de Biosfera Yabotí. Documento Técnico. Misiones, Argentina.

Ministerio de Hacienda de la Nación. 2018. Informes Productivos Provinciales. Misiones. Año 3 № 19. Buenos Aires. Argentina.

Miquelarena, A., and H. López. 2004. Considerations on the ichthyofauna of the Uruguay River basin: Hemiancistrus fuliginosus Cardoso y Malabarba, 1999 (Loricariidae: Ancistrinae). Journal of Applied Ichthyology-zeitschrift Fur Angewandte Ichthyologie 20:234-237. https://doi.org/10.1111/j.1439-0426.2004.00502.x.

Mirande, J. C., and S. Koerber. 2020. Checklist of the Freshwater Fishes of Argentina. 2nd edition. (CLOFFAR-2). Ichthyological Contributions of Peces Criollos 72:1-81

Munné, A., C. Solá, and N. Prat. 1998. QBR: Un índice rápido para la evaluación de la calidad de los bosques de ribera. Tecnología del Agua 175:20-37.

Munné, A., N. Prat, C. Solà, N. Bonada, and M. Rieradevall. 2003. A simple field method for assessing the ecological quality of riparian habitat in rivers and streams: QBR index. Aquatic Conservation: Maritime and Freshwater Ecosystems 13:147-163. https://doi.org/10.1002/aqc.529.

Myers, N., R. Mittermeier, C. Mittermeier, G. da Fonseca, and J. Kent. 2000. Biodiversity hotspots for conservation priorities. Nature 403:853-858. https://doi.org/10.1038/35002501.

Nel, L., D. Roux, G. Maree, C. Kleynhans, J. Moolman, B. Reyers, M. Rouget, and R. Cowling. 2007. Rivers in peril inside and outside protected areas: a systematic approach to conservation assessment of river ecosystems. Diversity and Distributions 13:341-352. https://doi.org/10.1111/j.1472-4642.2007.00308.x.

Nelson, J., T. Grande, and M. Wilson. 2016. Fishes of the World. Fifth Edition. Editorial John Wiley and Sons. New Jersey, USA. https://doi.org/10.1002/9781119174844.

Ongley, E. D. 1997. Lucha contra la contaminación agrícola de los recursos hídricos. (Estudio FAO Riego y Drenaje-55). GEMS/Water Collaborating Center Canada Center for Inland Waters. Pp. 21-37.

Oyakawa, O., and G. Mattox. 2009. Revision of the Neotropical trahiras of the Hoplias lacerdae species-group (Ostariophysi: Characiformes: Erythrinidae) with descriptions of two new species. Neotropical Ichthyology 7:117-140. https://doi.org/ 10.1590/S1679-62252009000200001.

Pacific Rivers Council. 2007. Freshwater Ecosystems in Crisis: A Synposis of Decline. Eugene: Pacific Rivers Council. Pp. 30.

Piálek, L., O. Rican, J. Casciotta, A. Almirón, and J. Zrzavy. 2011. Multilocus phylogeny of Crenicichla (Teleostei: Cichlidae), with biogeography of the C. lacustris group: species flocks as a model for sympatric speciation in rivers. Molecular Phylogenetics and Evolution 62:46-61. https://doi.org/10.1016/j.ympev.2011.09.006.

Piálek, L., K. Dragová, J. Casciotta, A. Almirón, and O. Říčan. 2015. Description of two new species of Crenicichla (teleostei: cichlidae) from the Lower Iguazú River with a taxonomic reappraisal of C. iguassuensis, C. tesay and C. yaha. Historia Natural, Tercera Serie 5:5-27.

Piálek, L., E. Burress, K. Dragová, A. Almirón, J. Casciotta, and O. Říčan. 2019. Phylogenomics of pike cichlids (Cichlidae: Crenicichla) of the $C$. mandelburgeri species complex: rapid ecological speciation in the Iguazú River and high endemism in the Middle Paraná basin. Hydrobiologia 832:355-375. https://doi.org/10.1007/s10750-018-3733-6.

Placci, G., and M. Di Bitetti. 2005. Situación Ambiental en la Ecorregión del Bosque Atlántico del Alto Paraná (Selva Paranaense). Pp. 197-210 en Galindo-Leal and Gusmão Câmara (eds.). Mata Atlántica Biodiversidad, Amenazas y Perspectivas. Fundación SOS Mata Atlántica, Belo Horizante, Brazil.

Reis, R., S. Kullander, and C. Ferraris Jr. 2003. Check list of the freshwater fishes of South Central and America. Porto Alegre: EDIPURCS.

Říčan, O., L. Piálek, A. Almirón, and J. Casciotta. 2011. Two new species of Australoheros (Teleostei: Cichlidae), with notes on diversity of the genus and biogeography of the Río de la Plata basin. Zootaxa 2982:1-26.

Říčan, O., A. Almirón, and J. Casciotta. 2017. Rediscovery of Crenicichla yaha (Teleostei: Cichlidae). Ichthyological Contributions of Peces Criollos 50:1-8.

Říčan, O., Š. Říčanová, K. Dragová, L. Piálek, A. Almirón, and J. Casciotta. 2018. Species diversity in Gymnogeophagus (Teleostei: Cichlidae) and comparative biogeography of cichlids in the Middle Paraná basin, an emerging hotspot of fish endemism. Hydrobiologia 832:331-354. https://doi.org/10.1007/s10750-018-3691-z.

Rodríguez, M., A. Cardozo, M. Ruiz Díaz, and D. Prado. 2005. Los bosques nativos misioneros: estado actual de su 
conocimiento y perspectivas. Pp. 3-33 en M. Arturi, J. Frangi and J. F. Goya (eds.). Ecología y Manejo de los bosques de Argentina. EDULP, La Plata, Argentina.

Romdhane, M. B., L. Riahi, B. Bouamama, H. Houissa, A. Ghorbel, and N. Zoghlami. 2018. Conservation priorities for endangered coastal North African Pennisetum glaucum L. landrace populations as inferred from phylogenetic considerations and population structure analysis. Heredity 122:219-232. https://doi.org/10.1038/s41437-018-0091-0.

Sabino, J., and J. Zuanon. 1998. A stream fish assemblage in Central Amazonia: distribution, activity patterns and feeding behavior. Ichthyological Exploration of Freshwaters 8:201-210.

Sarkar, S., and P. Illoldi-Rangel. 2010. Systematic conservation planning: an updated protocol. Natureza and Conservação 8:19-26. https://doi.org/10.4322/natcon.00801003.

Secretaría de Minería de la Nación. 2012. Sistema de información Geológica Ambiental Minera-SIGAM. URL: mineria.gov.ar/estudios/irn/misiones/m-3.asp.

SiFAP. 2019. Informe Nacional Ambiente y Áreas Protegidas de la Argentina. Sistema Federal de Áreas Protegidas. Secretaria de Ambiente y Desarrollo Sustentable, Argentina.

Srur, M. 2006. Identificación, caracterización y mapeo de los ambientes del Parque Nacional Iguazú. Informe técnico. Administración de Parques Nacionales. Buenos Aires Argentina.

Strayer, D., and D. Dudgeon. 2010. Freshwater biodiversity conservation: recent progress and future challenges. Journal of the North American Benthological Society 29:344-358. https://doi.org/10.1899/08-171.1.

Terán, G., J. Ferrer, M. Benitez, F. Alonso, G. Aguilera, and J. Mirande. 2017. Viviendo en las cascadas: Una nueva especie de Trichomycterus (Siluriformes: Trichomycteridae) del arroyo Tabay, Misiones, Argentina. PLoS ONE 12 (6): e190081. https://doi.org/10.1371/journal.pone.0179594.

Vane-Wright, R., C. Humphries, and P. Williams. 1991. What to protect? Systematics and the agony of choice. Biological Conservation 55:235-254. https://doi.org/10.1016/0006-3207(91)90030-D.

Vogler, R. E., A. Beltramino, E. Strong, J. Peso, and A. Rumi. 2015. A phylogeographical perspective on the ex situ conservation of Aylacostoma (Thiaridae, Gastropoda) from the High Paraná River (Argentina-Paraguay). Zoological Journal of the Linnean Society 174:487-499. https://doi.org/10.1111/zoj.12250.

WWF. 2010. Living Planet Report 2010 Biodiversity, biocapacity and development. Switzerland. 